\title{
NONCANCELLATIVE CONGRUENCES ON $N$-SEMIGROUPS ${ }^{1}$
}

\author{
ROBERT P. DICKINSON, JR.
}

\begin{abstract}
This paper describes a constructive method of finding all congruences $\rho$ on commutative cancellative archimedean semigroups $S$ without idempotents ( $N$-semigroups) such that $S / \rho$ is noncancellative and without idempotents. These congruences are refinements of $N$-congruences; a congruence $\eta$ is an $N$-congruence if $S / \eta$ is an $N$-semigroup.
\end{abstract}

I. Introduction. A commutative semigroup $S$ is called archimedean if every element divides some positive integer power of any other element: if $a, b \in S$ then there exist $c, d \in S$ and positive integers $m, n$ such that $a^{m}=b c$ and $b^{n}=a d$. Archimedean semigroups are classified into four types: T1, a nil-semigroup; T2, an ideal extension of a nil-semigroup by a group; T3, $N$-semigroups, cancellative without idempotent; T4, noncancellative without idempotent. Let $S$ be an $N$-semigroup. This paper describes a way of constructing all congruences, $\rho$, on $S$ such that $S / \rho$ is a semigroup of type $\mathrm{T} 4$, or a $\mathrm{T} 4$ semigroup. Two motivations for this paper are as follows: first, to contribute to the study of describing all congruences on $\mathrm{N}$-semigroups; second, to give a method of generating examples of T4 semigroups.

Suppose $S$, an $N$-semigroup, is homomorphic by $f$ onto $T$, a T4 semigroup. Tamura [4] has proven that any T4 semigroup has a homomorphic image which is an $N$-semigroup. So suppose $T$ is homomorphic by $g$ onto $V$, an $N$-semigroup. Let $\rho$ be the congruence induced by

$$
f: \rho=\{(x, y) \in S \times S: f(x)=f(y)\} .
$$

Let $\eta$ be the congruence on $S$ induced by the composite $g \circ f, S / \eta \cong V$. We have proven at this point that if an $N$-semigroup $S$ has a T4 congruence $\rho$ then $S$ has a proper T3 congruence $\eta$ ( $\eta$ is not the identity relation on $S$ ). Notice that the T4 congruence $\rho$ is a refinement of the proper T3 congruence $\eta$, or $\rho \subset \eta \neq \iota$. Easily from this discussion, all T4 congruences on an $\mathrm{N}$-semigroup are found as refinements of proper T3

Presented to the Society, April 22, 1972; received by the editors January 24, 1972. AMS (MOS) subject classifications (1969). Primary 2093.

1 Work performed under the auspices of the U.S. Atomic Energy Commission. 
congruences, or $N$-congruences. Theorems $2.3,2.4$ of this paper prove that if an $N$-semigroup $S$ has a proper $N$-congruence then it has a T4 congruence, which is a refinement. Thus a necessary and sufficient condition for an $\mathrm{N}$-semigroup to have a T4 congruence is that it has a proper $N$-congruence.

Not every $N$-semigroup has a proper $N$-congruence. For example, let $S$ be the semigroup of positive integers under ordinary addition. If $\rho$ is a proper congruence on $S$, then it may easily be shown that $S / \rho$ is finite. $S$ has congruences of both types $\mathrm{T} 1$ and $\mathrm{T} 2$.

$N$-semigroups, $N$-congruences and T4 congruences on $N$-semigroups can be described in terms of abelian groups and congruences on them, the nonnegative integers under addition, and various functions from the groups to the nonnegative integers. The first two functions are due to Tamura [3], [6]; they describe $N$-semigroups and $N$-congruences. The third function is the subject of this paper, and it tells how to refine an $\mathrm{N}$ congruence into a $\mathrm{T} 4$ congruence. We now describe Tamura's representation of an $N$-semigroup.

THEOREM 1.1. Let $G$ be an abelian group and let $N$ be the set of all nonnegative integers. Let I be a function from $G \times G$ into $N$ which satisfies the following conditions:

(1) $I(g, h)=I(h, g)$ for all $g, h \in G$.

(2) $I(g, h)+I(g h, i)=I(g, h i)+I(h, i)$ for all $g, h, i \in G$.

(3) $I(e, e)=1$, where $e$ is the identity of $G$.

(4) For every $g \in G$ there exists a positive integer $n$ such that $I\left(g^{n}, g\right)>0$.

Define a binary operation on $S=N \times G$ by

$$
(m, g)(n, h)=(m+n+I(g, h), g h) .
$$

Then $S$ is an $N$-semigroup, and every $N$-semigroup has such a representation.

In this paper all $N$-semigroups shall have the representation $(G, I)$ of the last theorem. Conditions (1) through (4) of that theorem will be called the axioms for an $I$-function. In this paper $e$ will denote the identity element of $G$. In axiom (2) let $g=h=e$, and let $i$ be arbitrary. One gets that $I(e, i)=1$. This fact is often used. Next we informally describe $N$-congruences on $N$-semigroups.

Let $\eta$ be an $N$-congruence on the $N$-semigroup $S=(G, I)$. The congruence $\eta$ induces a congruence on $G, \sigma$, as follows: $g \sigma h$ if and only if there exists $(n, g),(m, h) \in S$ such that $(n, g) \eta(m, h)$. The congruence $\eta$ also induces a function $d$ from $\sigma$ into $Z$, the integers, defined as follows: suppose $g \sigma h$, then there exist $(n, g),(m, h) \in S$ and $(n, g) \eta(m, h)$; define 
$d(g, h)=m-n$. This function is well defined and will satisfy conditions which we call $d$-axioms. These remarks are formally stated in the following theorem.

THEOREM 1.2. If $\eta$ is an $N$-congruence on $S=(G, I)$, then a congruence $\sigma$ on $G$ and a function d, from $\sigma$ into $Z$, the integers, are determined satisfying the following conditions (the d-axioms):

(1) $d(g, h)=-d(h, g)$, if $g \sigma h$.

(2) $d(g, i)=d(g, h)+d(h, i)$, if $g \sigma h$ and $h \sigma i$.

(3) $d(i g, i h)=d(g, h)+I(h, i)-I(g, i)$, if $g \sigma h$ and $i \in G$.

Conversely, assume that a congruence $\sigma$ on $G$ and $a$ d-function on $\sigma$ are given. Define $\eta$ on $S$ by: $(m, g) \eta(n, h)$ if, and only if, $g \sigma h$ and $n-m=$ $d(g, h)$. Then $\eta$ is an $N$-congruence on $S$, and every $N$-congruence is obtained in this manner.

Suppose $\eta$ is an $N$-congruence on $S=(G, I)$. Let $\sigma$ be the induced congruence on $G$. Suppose $\sigma=\iota$, the identical relation. We shall now prove that $\eta=\iota$. From axiom (1) for $d$-functions $d(g, g)=-d(g, g)=0$. Now suppose $(n, g) \eta(m, h)$. Then $g=h$ and $m=n$, for $m-n=d(g, h)=0$. Easily then, an $N$-congruence $\eta$ is proper if and only if the induced congruence $\sigma$ on $G$ is proper. It should be remarked that ('), the universal relation, is considered proper unless of course $\iota=\omega$.

Finally, we are ready to describe a third function, $\phi$, on $G \times G$ which shall describe T4 congruences. Recall that this function shall show how to refine proper $N$-congruences.

Definition 1.3. Let $S=(G, I)$ be an $N$-semigroup with proper $N$ congruence $\eta$. Associate with $\eta$ the pair $(d, \sigma)$. By a $\phi$-function associated with $(d, \sigma)$ is meant a function $\phi$ from $\sigma$ into the nonnegative integers satisfying the following (the $\phi$-axioms):

(1) $\phi(g, h)-\phi(h, g)=d(h, g)$, if $g \sigma h$.

(2) (Transitivity). For any $g, h, i \in G$ with $g \sigma h \sigma i$, let $k$ and $j$ be the nonnegative integers such that $\min \{k, j\}=0$, and $\phi(h, g)+k=\phi(h, i)+j$. Then there exists a nonnegative integer $m$ such that $\phi(g, i)+m=$ $\phi(g, h)+k$ and $\phi(i, g)+m=\phi(i, h)+j$.

(3) (Compatibility). For all $g, h, i \in G$, where $g \sigma h$ one has that $\phi(g, h)+$ $I(i, g) \geqq \phi(i g, i h)$.

(4) (Reflexivity). For all $g \in G, \phi(g, g)=0$.

In the next section we shall prove four theorems about $\phi$-functions. The motivations for the axioms will reveal themselves within the proofs.

Terms not defined in this paper may be found in [1].

II. Congruences and $\phi$-functions. The first theorem describes how to find a $\phi$-function given a T4 congruence on an $N$-semigroup. Recall that 
a T4 semigroup $T$ always has a homomorphic image $V$ which is an $N$ semigroup. Explicitly, define $\eta$ on $T$ by: $x \eta y$ if and only if there exists $c \in T$ such that $c x=c y$. Then $\eta$ is a congruence, and $T / \eta$ is an $N$-semigroup. The congruence $\eta$ is the smallest $N$-congruence on $T$ in that it is contained in all other $N$-congruences on $T$. The congruence $\eta$ can be defined on any commutative semigroup, and it is the smallest cancellative congruence defined on that semigroup [2], [4].

THEOREM 2.1. Let $S=(G, I)$ be an $N$-semigroup with a T4 congruence $\rho$. Let $\eta$ be the smallest $N$-congruence on $S$ such that $\rho \subset \eta$ (this exists by the previous discussion). Associate with $\eta$ the pair $(d, \sigma)$, and define $\phi$ on $\sigma$ as follow's: $\phi(g, h)=\min \{n:(n, g) \rho(m, h)$ for some $m\}$. Then this function is a $\phi$-function.

Proof. We first prove that the group congruence $\mu$ induced by $\rho$ is equal to $\sigma$, where $g \mu h$ means there exist $n, m$ such that $(n, g) \rho(m, h)$. Since $\rho \subset \eta$, we have that $\mu \subset \sigma$. We now prove the reverse inclusion.

Suppose $g \sigma h$. From the above discussion on smallest cancellative congruences on $S / \rho$ we have: $[k, w][n, g] \rho[k, w][m, h]$, for some $k, n, m$, where $[n, g]$ denotes congruence class of $\rho$ containing $(n, g)$. Thus $[l, w g] \rho[j, w h]$ for some $l, j$, and $w g \mu w h$. Since $\mu$ is a group congruence, we have $w^{-1}(w g) \mu w^{-1}(w h)$, or $g \mu h$. Thus $\sigma \subset \mu$, or $\mu=\sigma$. Thus $\phi$ is defined on $\sigma$.

We now prove that axiom (1) holds. Suppose that $\phi(g, h)=m$. Then there exists $n$ such that

(a) $(m, g) \rho(n, h)$.

We shall show that $n=\phi(h, g)$. Then since $(m, g) \eta(n, h)$, (1) will be proven. Certainly, $k=\phi(h, g) \leqq n$. Assume $k<n$, and let $k+l=n$. We shall derive a contradiction. This will prove $k=n$. There exists $s$ such that:

(b) $(k, h) \rho(s, g)$, so $s \geqq \phi(g, h)$.

By compatibility for $\rho$ we have:

(c) $(l-1, e)(k, h) \rho(l-1, e)(s, g)$, or

(d) $(l+k, h) \rho(l+s, g)$.

Using the fact that $\rho \subset \eta$ and (a) and (d) we have:

(e) $n-m=d(g, h)=(l+k)-(l+s)=n-(l+s)$.

Thus $m=l+s$; since $s \geqq \phi(g, h)=m, l=0$, or $k=n$, which is the contradiction.

We now prove the second axiom. We shall assume $j=0$, the case $k=0$ being the same. Thus we have:

(f) $\phi(h, g)+k=\phi(h, i)$.

Suppose $k=0$. In this case, we have:

$$
(\phi(g, h), g) \rho(\phi(h, g), h)=(\phi(h, i), h) \rho(\phi(i, h), i) .
$$


By transitivity:

(g) $(\phi(g, h), g) \rho(\phi(i, h), i)$.

Thus $\phi(g, i) \leqq \phi(g, h)$, or there exists a nonnegative integer $m$ such that:

(h) $\phi(g, i)+m=\phi(g, h)+k$.

If $k>0$ the same argument is true by using compatibility with $(k-1, e)$ as multiplier. In either case then equation (h) is true.

We derive equation (i), which follows by substitution, using (f), (h) and using the fact that $\phi$ satisfies axiom (1).

(i) $\phi(i, g)+m=\phi(i, h)$.

Start by substituting into equation (f):

(j) $d(g, h)+\phi(g, h)+k=d(i, h)+\phi(i, h)$.

Using the first two axioms for $d$, replace $d(g, h)-d(i, h)$ with $d(g, i)$ :

(k) $d(g, i)+\phi(g, h)+k=\phi(i, h)$.

Now let $d(g, i)=\phi(i, g)-\phi(g, i)$, and use (h) to finally derive (i). This completes the proof of axiom (2).

We now prove that axiom (3) holds. In part one we proved:

(l) $(\phi(g, h), g) \rho(\phi(h, g), h)$.

Since $\rho$ is compatible, multiply both sides by $(0, i)$ to get :

(m) $(\phi(g, h)+I(i, g), i g) \rho(\phi(h, g)+I(i, h), i h)$.

Thus it follows that $\phi(g, h)+I(i, g) \geqq \phi(i g, i h)$.

The fourth axiom holds since trivially $(0, g) \rho(0, g)$. Q.E.D.

The next theorem allows one to construct a congruence from a $\phi$-function.

THEOREM 2.2. Let $S=(G, I)$ be an $N$-semigroup, and let $\eta$ be a proper $N$-congruence on $S$ with associated pair $(d, \sigma)$. Let $\phi$ be $a$-function associated with $(d, \sigma)$. Define $\rho$ on $S$ as follows: $(n, g) \rho(m, h)$ if, and only if:

(1) goh.

(2) There exists a nonnegative integer $k$ such that $n=\phi(g, h)+k$ and $m=\phi(h, g)+k$.

Then $\rho$ is a congruence on $S$. One easily notes that $\rho \subseteq \eta$.

Proof. Since $\phi(g, g)=0$ for all $g \in G$, it follows that $\rho$ is reflexive. Also, it is easy to see that $\rho$ is symmetric. We now prove transitivity.

Suppose $(n, g) \rho(m, h)$ and $(m, h) \rho(k, i)$. The following equations follow from the definition of $\rho$. Since $\rho \subseteq \eta, g \sigma i$.

(a) $n=\phi(g, h)+s$.

(b) $m=\phi(h, g)+s=\phi(h, i)+t, s, t \geqq 0$.

(c) $k=\phi(i, h)+t$.

Next we use (2) for $\phi$-functions:

(d) $\phi(h, g)+u=\phi(h, i)+v \min \{u, v\}=0$. 
There exists a nonnegative integer $" 1$ such that:

(e) $\phi(g, i)+w=\phi(g, h)+u$.

(f) $\phi(i, g)+w=\phi(i, h)+v$.

Using (a) and (e) we obtain $n=\phi(g, i)+\cdots-u+s$.

Using (c) and (f) we obtain $k=\phi(i, g)+w-v+t$.

Since $\min \{u, v\}=0$, it follows that $s \geqq u$, and $t \geqq v$ (subtract (b) and (d)). Thus $w-u+s$ and $u-v+t$ are nonnegative. We shall now show that these two expressions are equal. We do this by showing that $s-t=u-v$. These equations follow from (b) and (d). From what has now been proven, and the easy fact that $g \sigma i$, we have that $(n, g) \rho(k, i)$.

We now prove compatibility. Again we need that $\rho \subseteq \eta$. Suppose $(n, g) \rho(m, h)$. We must prove that $(k, i)(n, g) \rho(k, i)(m, h)$, where $(k, i)$ is an arbitrary member of $S$. More explicitly, we must show that $(k+n+I(i, g), i g) \rho(k+m+I(i, h), i h)$. This last pair is $\eta$ related, since $\eta$ is compatible. We must show the following:

$$
\begin{aligned}
k+n+I(i, g) & =\phi(i g, i h)+u, \quad u \geqq 0, \\
k+m+I(i, h) & =\phi(i h, i g)+u .
\end{aligned}
$$

Now since $(n, g) \rho(m, h)$, there exists $t \geqq 0$ such that $n=\phi(g, h)+t$ and $m=\phi(h, g)+t$. To complete the proof consider the following:

$$
\begin{aligned}
w & =k+\phi(g, h)+t+I(i, g)-\phi(i g, i h), \\
v & =k+\phi(h, g)+t+I(i, h)-\phi(i h, i g) .
\end{aligned}
$$

We show that $w=v$, and that $w \geqq 0$; of course $w=u$. Now $w$ is greater than or equal to zero by axiom (3) on $\phi$. To show $n=v$, form $w-v$, substitute $d$ expressions for $\phi$ differences, and then use axiom (3) for $d$ functions. Q.E.D.

Let $\eta$, with $(d, \sigma)$, be an $N$-congruence on an $N$-semigroup $S$. It is obvious that distinct $\phi$-functions associated with $\eta$ will produce distinct congruences $\rho$ as in Theorem 2.2. Also, let $\rho$ be a T4 congruence and $\eta$ the smallest $N$-congruence containing $\rho$. Let $\phi$ be determined as in Theorem 2.1, and let $\theta$ be determined from $\phi$ as in Theorem 2.2. Then $\rho=\theta$.

It may be noted that any congruence $\mu$ on $S$ induces a group congruence $\sigma$ on $G: g \sigma h$ if, and only if, $(n, g) \mu(m, h)$, for some $n, m$. Thus $\rho$ induces a group congruence $\sigma$. Also from the definition of $\phi$ in Theorem 2.1, we see that $\phi$ is only dependent upon $\rho$, and not upon any $\eta$ which might contain $\rho$. Although we do not follow this point, it is possible to define $d$-functions from T4-congruences and their induced group congruences. The produced $N$-congruence, $\eta$, will be contained in all $N$-congruences containing $\rho$.

Let $\rho$ be a refinement of $\eta$, an $N$-congruence on an $N$-semigroup $S$. 
Easily, $S / \rho$ is either an $N$-semigroup or a T4 semigroup, for $S / \rho$ is homomorphic onto $S / \eta$ which has no idempotent, hence $S / \rho$ has none. Not every $\phi$-function will cause a proper refinement of an $N$-congruence (see Example 2.5). The next theorem describes this situation.

THEOREM 2.3. Let $S=(G, I)$ be an $N$-semigroup with an $N$-congruence $\eta$. Let $\eta$ be associated with the pair $(d, \sigma)$, and let $\phi$ be a $\phi$-function associated with $(d, \sigma)$. A necessary and sufficient condition for $\phi$ to cause a refinement (as in Theorem 2.2) of $\eta$ is that there exist $g, h \in G$ such that $g \sigma h$ and $\phi(g, h) \geqq \phi(h, g) \geqq 1$. In this case the congruence induced by $\phi$ is T4.

Proof. Let $\rho$ be the induced congruence by $\phi$. Easily, $\rho \subseteq \eta$. We now prove sufficiency. Assume there exists $g, h \in G$ such that $\phi(g, h) \geqq \phi(h, g) \geqq$ 1. Then $(\phi(g, h)-1, g) \eta(\phi(h, g)-1, h)$, but this pair is not $\rho$ related. (In a sense $\phi$-functions determine a threshold for refinement.) Thus it has been proven that $\rho \neq \eta$.

In this case $S / \rho$ is not cancellative, for $(\phi(g, h)-1, g) \phi(\phi(h, g)-1, h)$, but $(0, e)(\phi(g, h)-1, g)=(\phi(g, h), g) \rho(\phi(h, g), h)=(0, e)(\phi(h, g)-1, h)$. Thus in $S / \rho$ one has that $a x=a y$ but $x \neq y$.

We now prove necessity by proving the contrapositive; in this case if $g \sigma h$ then either $\phi(g, h)=0$, or $\phi(h, g)=0$. We now prove that $\rho=\eta$. Suppose that $(n, g) \eta(m, h)$. Then $m-n=d(g, h)=\phi(h, g)-\phi(g, h)$ and $g \sigma h$. If $d(g, h)=0$, then $m=n$, and $\phi(h, g)=\phi(g, h)=0$. Thus $(n, g) \rho(m, h)$. Suppose $d(g, h)>0$. Then we must have that $\phi(g, h)=0$. Thus $m-n=$ $\phi(h, g)$, or $m=\phi(h, g)+n$; also, $n=\phi(g, h)+n$. Thus $(n, g) \rho(m, h)$. The case where $d(g, h)<0$ is similar. Q.E.D.

The following theorem shows that if an $N$-semigroup has a proper $N$-congruence $\eta$ then $\eta$ has an infinite number of refinements which are T4-congruences. These refinements form a chain with respect to inclusion.

THEOREM 2.4. Let $S=(G, I)$ be an $N$-semigroup with a proper $N$ congruence $\eta$, where as usual $\eta$ has the associated pair $(d, \sigma)$. Define $\phi$ on $(d, \sigma)$ by:

$$
\begin{aligned}
\phi(g, g) & =0, & & \\
\phi(g, h) & =d(h, g)+m & & \text { if } d(h, g) \geqq 0, \\
& =m & & \text { otherwise. }
\end{aligned}
$$

Then $\phi$ is $a$-function for each $m=0,1,2, \cdots$.

Proof. To verify the first two axioms for $\phi$-functions we only need the first two axioms for $d$-functions: (1) $d(g, h)=-d(h, g) ;(2) d(g, i)=$ $d(g, h)+d(h, i)$. 
We now verify that $\phi(g, h)-\phi(h, g)=d(h, g)$. Consider three cases: (1) $d(g, h)>0$; (2) $d(g, h)=0$; (3) $d(g, h)<0$. We only prove case (1). In this case $m-(d(g, h)+m)=-d(g, h)=d(h, g)$.

We now verify the second axiom. Suppose the following:

(A) $\phi(h, g)+k=\phi(h, i)+j$, where $\min \{k \cdot j\}=0$.

We must find a nonnegative integer $t$ such that $\phi(g, i)+t=\phi(g, h)+k$ and $\phi(i, g)+t=\phi(i, h)+j$. Let $u=\phi(g, h)+k-\phi(g, i)$, and let $v=\phi(i, h)+$ $j-\phi(i, g)$. We shall prove two things which complete the proof: (1) $u=v$; (2) $u \geqq 0$. Of course $u=t$. We now prove (1).

(a) $d(i, h)+d(h, g)=d(i, g)$.

(b) $d(h, g)+\phi(h, i)-\phi(i, h)=\phi(g, i)-\phi(i, g)$.

(c) $d(h, g)+\phi(h, i)+j-\phi(g, i)=\phi(i, h)+j-\phi(i, g)$.

(d) $d(h, g)+\phi(h, g)+k-\phi(g, i)=v$.

(e) $u=\phi(g, h)+k-\phi(g, i)=v=\phi(i, h)+j-\phi(i, g)$.

Step (d) follows from (c) by using (A). We now prove (2).

$$
t=u=\phi(g, h)+k-\phi(g, i)=\phi(i, h)+j-\phi(i, g) .
$$

Simply note that $t \geqq 0$ because either $\phi(g, i)=m$, or $\phi(i, g)=m$, and $\phi(x, y) \geqq m, x \neq y$. The proof is trivial if $g, h, i$ are not distinct. We now prove the third axiom.

One must prove the following:

(B) $\phi(g, h)+I(i, g) \geqq \phi(i g, i h)$.

Multiply (3) in the axioms of the $d$-function by -1 ; then use (1) of those axioms to produce the following:

$$
d(h, g)-I(h, i)+I(g, i)=d(i h, i g) .
$$

Since the $I$-function is nonnegative and symmetric one obtains:

(C) $d(h, g)+I(i, g) \geqq d(i h, i g)$.

Using (C) with the various substitutions one can make for $\phi$ in (B), one easily proves the desired result. For example, suppose $d(h, g)<0$ and $d(i h, i g)>0$. By $(\mathrm{C})$ one has that $I(i, g)>d(i h, i g)$. Substituting for $\phi$ in (B) gives: $m+I(i, g) \geqq d(i h, i g)+m$. Q.E.D.

EXAMPLE 2.5. Let $N$ be the positive integers under addition, and let $C_{2}=\{e, a\}$ be the cyclic group of order two. Then $S=N \times C_{2}$, the direct product, is an $N$-semigroup. $S$ may be represented by the pair $\left(C_{2}, I\right)$, where $I(x, y)=1$. The map $f$ such that $f(n, g)=(n-1, g)$ is an isomorphism between the two representations, where the domain is $N \times C_{2}$. Let $\pi$ be the projection of $S$ onto $N$. The induced congruence $\sigma$ on $C_{2}$ is $\omega$, the universal relation. The induced $d$-function is identically zero. In Theorem 2.4 , If we took $\phi$ with $m=0$, we would get $\phi$ identically zero. 
EXAMPLE 2.6. This example shows that not every $\phi$-function associated with $(d, \sigma)$ is found as in Theorem 2.4. The $N$-semigroup $S$ is defined on $C_{3}$, the group of order 3 .

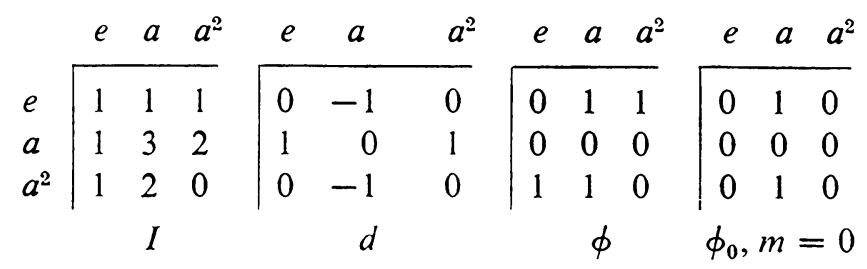

Here $\sigma=\omega$ on $C_{3}$.

In conclusion, we have shown that an $N$-semigroup $S$ can have a T4congruence if and only if it has a proper $N$-congruence. We have shown that all T4-congruences are refinements of proper $N$-congruences, and every proper $N$-congruence has such a refinement. Using the $\phi$-function we have established a connection between $(d, \sigma)$ representations of $N$ congruences and T4-congruences.

\section{REFERENCES}

1. A. H. Clifford and G. B. Preston, The algebraic theory of semigroups. Vol. I, Math. Surveys, no. 7, Amer. Math. Soc., Providence, R.I., 1961. MR 24 \#A2627.

2. R. P. Dickinson, Jr., On right zero unions of commutative semigroups, Pacific J. Math. 41 (1972).

3. T. Tamura, Commutative nonpotent archimedean semigroup with cancellation law. I, J. Gakugei Tokushima Univ. 8 (1957), 5-11. MR 20 \#3224.

4. - Construction of trees and commutative archimedean semigroups, Math. Nachr. 36 (1968), 257-287. MR 37 \#6222.

5. - Abelian groups and R-semigroups, Proc. Japan Acad. 46 (1970), 212-216. MR 43 \#381.

6. - $N$-congruences on $N$-semigroups (to appear).

Computation Department, Lawrence Radiation Laboratory, University of California, Livermore, California 94550 